\title{
Quad-Band Bowtie Antenna Design for Wireless Communication System Using an Accurate Equivalent Circuit Model
}

\author{
Mohammed Moulay, ${ }^{1}$ Mehadji Abri, ${ }^{1}$ and Hadjira Abri Badaoui ${ }^{2}$ \\ ${ }^{1}$ Telecommunications Laboratory, University of Tlemcen, Tlemcen, Algeria \\ ${ }^{2}$ STIC Laboratory, Faculty of Technology, University of Tlemcen, Tlemcen, Algeria \\ Correspondence should be addressed to Mehadji Abri; abrim2002@yahoo.fr
}

Received 22 September 2014; Revised 19 December 2014; Accepted 22 December 2014

Academic Editor: Giancarlo Bartolucci

Copyright (c) 2015 Mohammed Moulay et al. This is an open access article distributed under the Creative Commons Attribution License, which permits unrestricted use, distribution, and reproduction in any medium, provided the original work is properly cited.

\begin{abstract}
A novel configuration of quad-band bowtie antenna suitable for wireless application is proposed based on accurate equivalent circuit model. The simple configuration and low profile nature of the proposed antenna lead to easy multifrequency operation. The proposed antenna is designed to satisfy specific bandwidth specifications for current communication systems including the Bluetooth (frequency range 2.4-2.485 GHz) and bands of the Unlicensed National Information Infrastructure (U-NII) low band (frequency range 5.15-5.35 GHz) and U-NII mid band (frequency range 5.47-5.725 GHz) and used for mobile WiMAX (frequency range 3.3-3.6 GHz). To validate the proposed equivalent circuit model, the simulation results are compared with those obtained by the moments method of Momentum software, the finite integration technique of CST Microwave studio, and the finite element method of HFSS software. An excellent agreement is achieved for all the designed antennas. The analysis of the simulated results confirms the successful design of quad-band bowtie antenna.
\end{abstract}

\section{Introduction}

The world of wireless telecommunications evolves rapidly. Wireless networking represents the future of computer and Internet connectivity worldwide. This technology enables two or more computers to communicate using standard network protocols. Broadband wireless technologies are increasingly gaining popularity by the successful global deployment of the Wireless Personal Area Networks WLAN in IEEE 802.11 a/b, Wireless Metropolitan Area Networks (WiMAX-IEEE 802.16a), and bands of the Unlicensed National Information Infrastructure (U-NII). Wireless networks provide all of the features and benefits of traditional local area network technologies such as Ethernet and Token Ring without the limitations of wires and cables.

Recently, WLANs have gained a strong popularity and great place in the local area network (LAN) market. Today, WLANs based on the IEEE 802.11 standard are considered a practical and interesting solution of network connection offering mobility, flexibility, and low cost of deployment and use. The IEEE 802.11a operates at three bands. The first band extends from 5.15 to $5.25 \mathrm{GHz}$, the second from 5.25 to $5.35 \mathrm{GHz}$, and the third from 5.725 to $5.825 \mathrm{GHz}$. Unlicensed National Information Infrastructure (U-NII) band is used in WLAN, Bluetooth, and Wi-Fi operations. The U-NII band can be divided into three subbands as U-NII low band (frequency range $5.15-5.35 \mathrm{GHz}$ ), U-NII mid band (frequency range $5.47-5.725 \mathrm{GHz}$ ), and U-NII high band (frequency range $5.725-5.875 \mathrm{GHz}$ ) [1-5].

Recently, wireless communication for wireless local area network (WLAN) and Worldwide Interoperability for Microwave Access (WiMAX) have experienced tremendous growth [6].

Antennas design for wireless communication systems has attracted a great interest during the last years [7, 8]. Printed antennas have a number of advantages, such as low profile, light weight, and low cost, which make them perfect to be used in wireless communication applications, and ease 


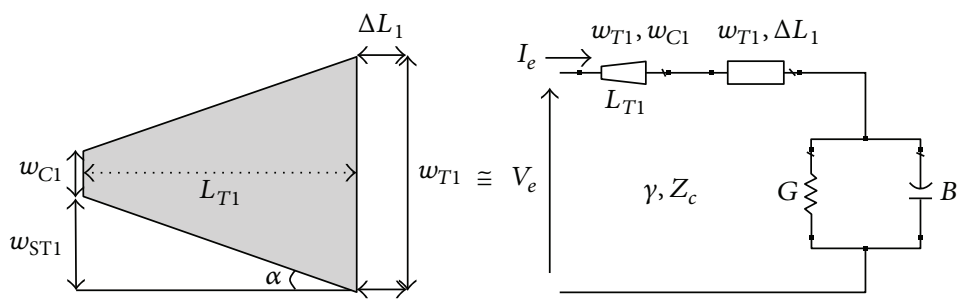

(a)

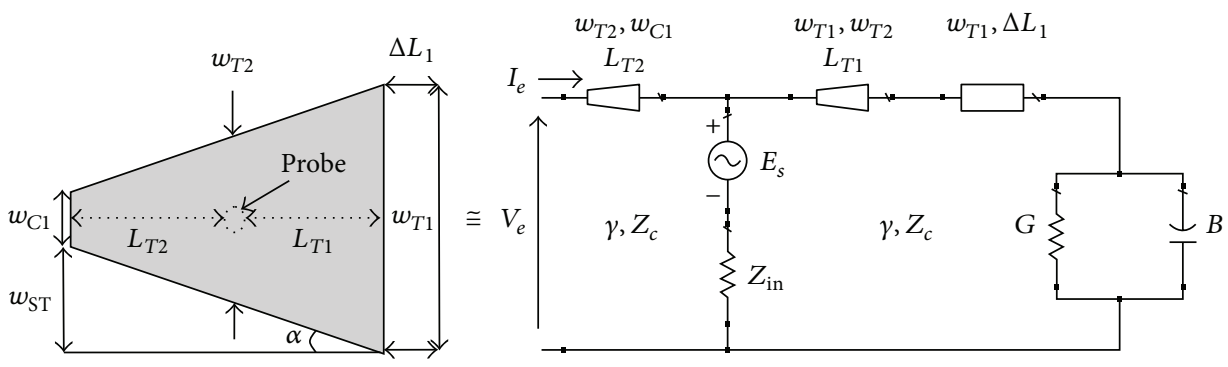

(b)

Figure 1: (a) Configuration of one taper bowtie antenna and the corresponding equivalent circuit model. (b) Configuration of bowtie antenna fed by coaxial probe and the corresponding equivalent circuit model.

of integration with microwave circuits [9-13]. A multiband antenna is attractive in many commercial applications as it is designed to have a single radiator with a capability to transmit and receive multiple frequencies. Nevertheless, a multiband antenna may not sufficiently cover the required operating bands. Therefore, an antenna which is able to operate with multiple independent frequency bands is required. This antenna should also provide ease in controlling the desirable resonance frequencies, impedance bandwidths, radiation patterns, and polarizations. These are obviously becoming the most important factors for the applications of antennas in both contemporary and future wireless communication systems.

Rigorous numerical methods such as integral equations solved by the method of moments are preferable to analyze such bowtie antenna structures. These methods offer better accuracy, but they require long and tedious calculations. As a result, these methods cannot be used in optimization. The equivalent circuit model is perfectly suited to this type of antennas.

In this paper, we propose a novel quad-band bowtie antenna covering the operating band of Bluetooth operating in the range frequency $(2.40-2.484 \mathrm{GHz})$, two bands of the Unlicensed National Information Infrastructure (UNII) low band (frequency range $5.15-5.35 \mathrm{GHz}$ ) and U-NII mid band (frequency range $5.47-5.725 \mathrm{GHz}$ ), and mobile Worldwide Interoperability for Microwave Access (WiMAX) operating in the range frequency $(3.40-3.60 \mathrm{GHz})$. The proposed antenna was modeled and optimized using the proposed equivalent circuit model and the obtained results are compared with those given by moments method of Agilent software, the finite integration technique of the CST Microwave studio, and the finite element method of HFSS software. By adjusting the radiating elements dimensions and adding other elements or tapers, broad bandwidth behavior suitable for Bluetooth, mobile WiMAX, and two bands of the Unlicensed National Information Infrastructure (U-NII) can be achieved. Details of the antenna design results are presented and discussed.

\section{Monoband, Dual-Band, and Quad-Band Bowtie Antenna Equivalent Circuit}

2.1. Monoband Bowtie Antenna Equivalent Circuit. In this section, the equivalent circuit applied to bowtie antenna is presented. This model represents the patch as a lowimpedance microstrip line whose width determines the impedance and effective dielectric constant. A combination of parallel-plate radiation conductance and capacitive susceptance loads both radiating edges of the patch. This simple circuit is used directly from the transmission line model to compute the input impedance matching at any frequency providing the resonance. The geometry of one taper of the bowtie microstrip antenna and those fed by coaxial probe are shown in Figure 1.

$L_{T 1}$ represents the length, $w_{T 1}$ is the open end width, and $w_{C 1}$ is the width at the input of the structure. $\Delta L$ is the physical length of the radiating slot which forms a useful model for calculating the radiation field of the antenna. It is given by the following formula [14-18]:

$$
\Delta L=h \frac{0.412\left(\varepsilon_{\mathrm{eff}}+0.3\right)\left(w_{T 1} / h+0.264\right)}{\left(\varepsilon_{\mathrm{eff}}-0.258\right)\left(w_{T 1} / h+0.8\right)} .
$$

$\varepsilon_{\text {eff }}$ is the corresponding effective dielectric constant:

$$
\varepsilon_{\text {eff }}=\frac{1+\varepsilon+(\varepsilon-1) / \sqrt{1+10\left(h / w_{T 1}\right)}}{2} .
$$




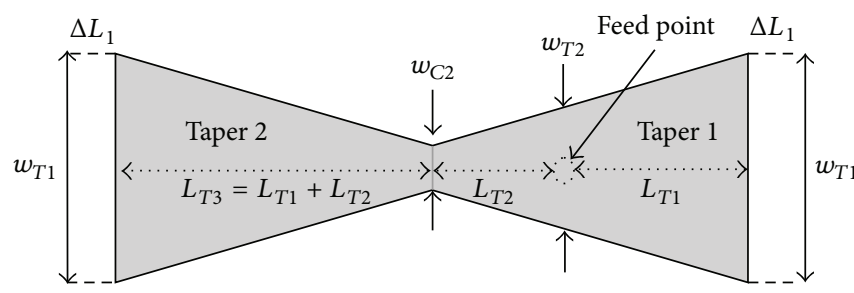

(a)

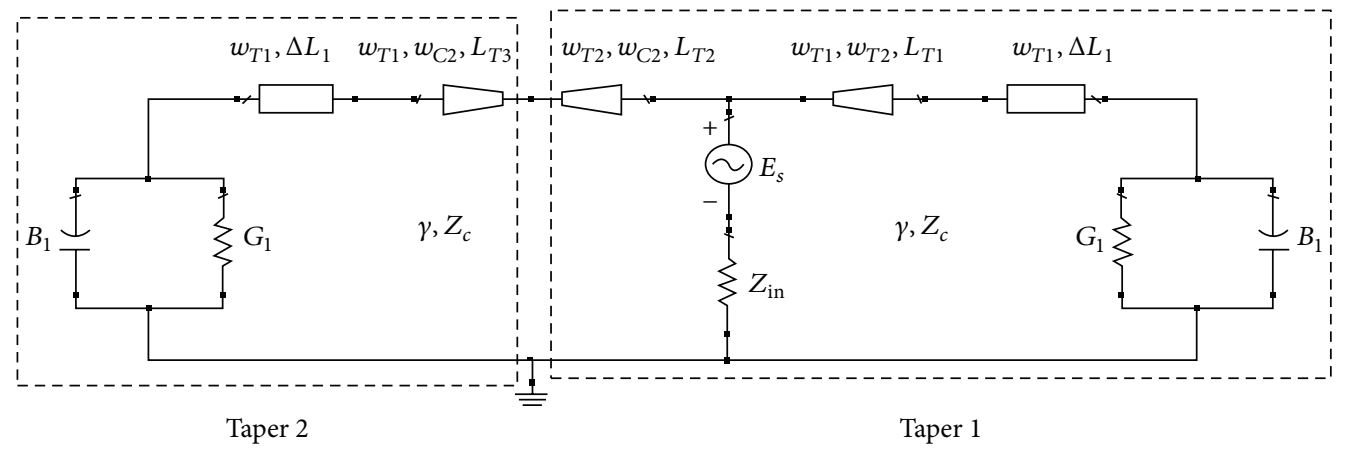

(b)

Figure 2: (a) Configuration of the proposed monoband bowtie antenna composed of two tapers with same dimension fed by coaxial probe. (b) The corresponding equivalent circuit.

$\gamma$ is the constant propagation and $Z_{c}$ is the characteristic impedance. It is given by the following rigorous formula:

$$
\begin{gathered}
Z_{c}=120 \pi\left(2 \sqrt { \varepsilon _ { \mathrm { eff } } } \left(\frac{w_{T 1}}{2 h}+0.082\left(\frac{\left(\varepsilon_{\mathrm{eff}}-1\right)}{\varepsilon_{\mathrm{eff}}^{2}}\right)\right.\right. \\
\left.\left.+\varepsilon_{\mathrm{eff}}+0.411\right)\right)^{-1} .
\end{gathered}
$$

$B$ and $G$ are capacitive and conductive components of the edge admittance $Y$. The susceptance $B$ accounts for the fringing field associated with the radiating edge of the width $w_{T 1}$, and $G$ is the conductance contributed by the radiation field associated with each edge. Each radiating slot is represented by an equivalent parallel admittance $(Y)$ [17]. The equivalent width of the slots of width $w_{\mathrm{ST} 1}$ is calculated using the following formula:

$$
w_{\mathrm{ST} 1}=\frac{\left(w_{T 1}-w_{\mathrm{C} 1}\right)}{2}=L_{T 1} \tan \alpha
$$
below.

The expressions of $G$ and $B$ are given by the relations

The angle $\alpha$ must not exceed $10^{\circ}$ and $w_{T 1}$ must be less than $2 \mathrm{~mm}$ in optimization operation for the results accuracy [4]. Consider

$$
G=\frac{60(\pi h)^{2}}{Z_{c}^{2} \lambda^{2}}\left[\frac{\left(\varepsilon_{\mathrm{eff}}+1\right)}{\varepsilon_{\mathrm{eff}}}-\frac{\left(\varepsilon_{\mathrm{eff}}-1\right)^{2}}{2 \varepsilon_{\mathrm{eff}} \sqrt{\varepsilon_{\mathrm{eff}}}} \cdot \log \left(\frac{\sqrt{\varepsilon_{\mathrm{eff}}}+1}{\sqrt{\varepsilon_{\mathrm{eff}}}-1}\right)\right],
$$

where $\lambda$ is the wavelength and $h$ is height of the substrate.
$V_{e}$ and $I_{e}$ are, respectively, the voltage and current of the source.

When we associate the two tapers, a bowtie antenna is obtained with two radiating elements. Figure 2 shows the structure of the monoband bowtie antenna and its equivalent circuit fed by coaxial probe designed for single resonance, with double radiating elements with same sizes.

2.2. Dual-Band Bowtie Antenna Equivalent Circuit. The dualband bowtie antenna fed by coaxial probe designed to operate in two resonant frequencies and its equivalent circuit are shown in Figure 3. The two tapers are of different dimensions in order to allow operation at different frequencies.

2.3. Quad-Band Bowtie Antenna Equivalent Circuit. In this section, an equivalent circuit model for quad-band bowtie antenna design is proposed. In order to allow the antenna to resonate at multiple frequencies, it is necessary to add several radiating elements. The quad-band bowtie antenna consists of four tapers with different sizes; each taper radiates at a specific resonance frequency. The configuration of the proposed quad-band bowtie antenna and its corresponding equivalent circuit model are depicted in Figure 4.

\section{Simulation Results}

To test the validity of the proposed equivalent circuit model for the bowtie antenna, several simulations were made and compared with those obtained by the moments method and the finite integration technique. The proposed optimized bowtie antennas are designed on an FR4 substrate with thickness $1.6 \mathrm{~mm}$ and relative permittivity 4.32 and a loss tangent of about 0.048 and $0.05 \mathrm{~mm}$ conductor thickness. 


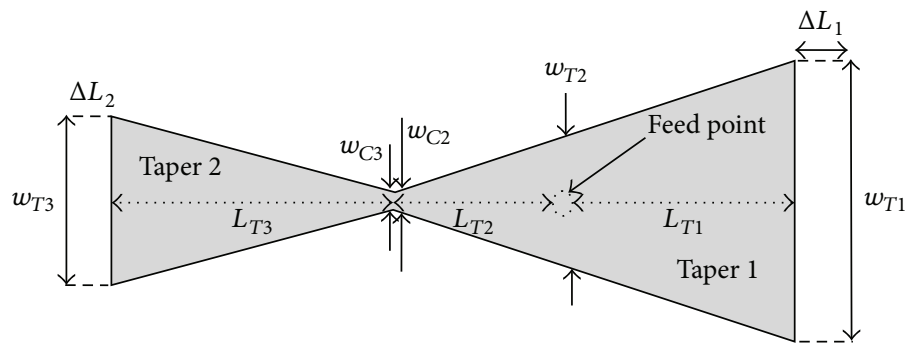

(a)

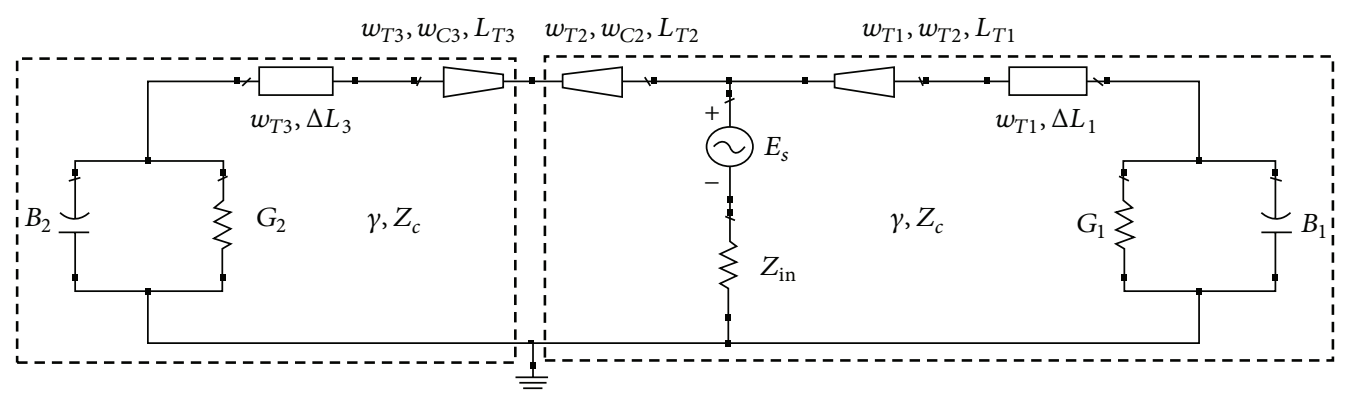

Taper 2

Taper 1

(b)

FIgURE 3: (a) Configuration of the proposed dual-band bowtie antenna with two tapers of different dimension fed by coaxial probe. (b) The corresponding equivalent circuit.

3.1. Single Band Bowtie Antenna for Bluetooth Applications. The reflection coefficient of monoband bowtie antenna is displayed from 2 to $3 \mathrm{GHz}$ in Figure 5. The mask layout of bowtie antenna operating at centered frequency $2.44 \mathrm{GHz}$ for Bluetooth applications is presented in the same figure.

Let us notice that the simulated reflection coefficient of the antenna is found below $-20 \mathrm{~dB}$ at the desired frequency. An excellent agreement is observed between the proposed equivalent circuit model, the moments method, and the finite integration technique. The beam width is well covered with the three models. Notice that a peak of $-33 \mathrm{~dB}$ at the resonant frequency is obtained by the transmission line model, of about $-32.5 \mathrm{~dB}$ by, respectively, CST Microwave studio and HFSS software and $-23 \mathrm{~dB}$ by the moments method of ADS software.

3.2. Dual-Band Bowtie Antenna. The antenna was simulated using the dual equivalent circuit and the results are compared with those obtained by the moments method and the finite integrated technique based on CST Microwave studio. The antenna was optimized to operate at $2.44 \mathrm{GHz}$ and $5.6 \mathrm{GHz}$, respectively, for Bluetooth and U-NII mid bands. Figure 6 shows the simulated reflection coefficient versus frequency for the proposed dual-band bowtie antenna consisting of two tapers with different sizes.

Notice that a perfect concordance is achieved between the proposed equivalent circuit model, Momentum, CST, and HFSS software. It can be observed that the simulated two resonant frequencies $2.44 \mathrm{GHz}$ and $5.6 \mathrm{GHz}$ give a good impedance matching. The simulated reflection coefficients are below $-10 \mathrm{~dB}$ in the bandwidth range from $2.4 \mathrm{GHz}$ to
$2.50 \mathrm{GHz}$ with respect to the center frequency at $2.44 \mathrm{GHz}$, and also in the bandwidth range from $5.45 \mathrm{GHz}$ to $5.725 \mathrm{GHz}$ with respect to the center frequency at $5.69 \mathrm{GHz}$. These bands are practically wide bands and located at the Bluetooth and U-NII mid band.

3.3. Quad-Band Bowtie Antenna. In this section, numerical results of a quad-band printed bowtie antenna are presented. The geometry of the designed quad-band bowtie antenna consists of four tapers and is designed on the same substrate as the previous sections. The excellent performance of this bowtie antenna gives us the advantage that by modifying its structural parameters and by adding other resonant elements we can obtain several frequency bands for this antenna by introducing a proper optimization. A comparison of reflection coefficient obtained with the equivalent model, CST Microwave studio, Momentum, and HFSS is displayed in Figure 7 in the range frequency $(2-6 \mathrm{GHz})$.

From the results shown in Figure 7, four resonances are observed and excellent coherence between the simulated results is observed. The equivalent circuit results are very similar to those simulated by Momentum, CST Microwave studio, and HFSS in all the range frequency. A very small shift between the simulations at the fourth band is recorded. The antenna can generate four operating bands to, respectively, cover the Bluetooth band, mobile WiMAX, and the Unlicensed National Information Infrastructure (U-NII) with two bands. The first resonance occurs at $f_{1}=2.44 \mathrm{GHz}$, the second resonance occurs at $f_{2}=3.5 \mathrm{GHz}$, the third resonance occurs at $f_{3}=5.25 \mathrm{GHz}$, and the fourth resonance occurs at $f_{4}=5.6 \mathrm{GHz}$. Let us notice the appearance of some pics at 3.1 


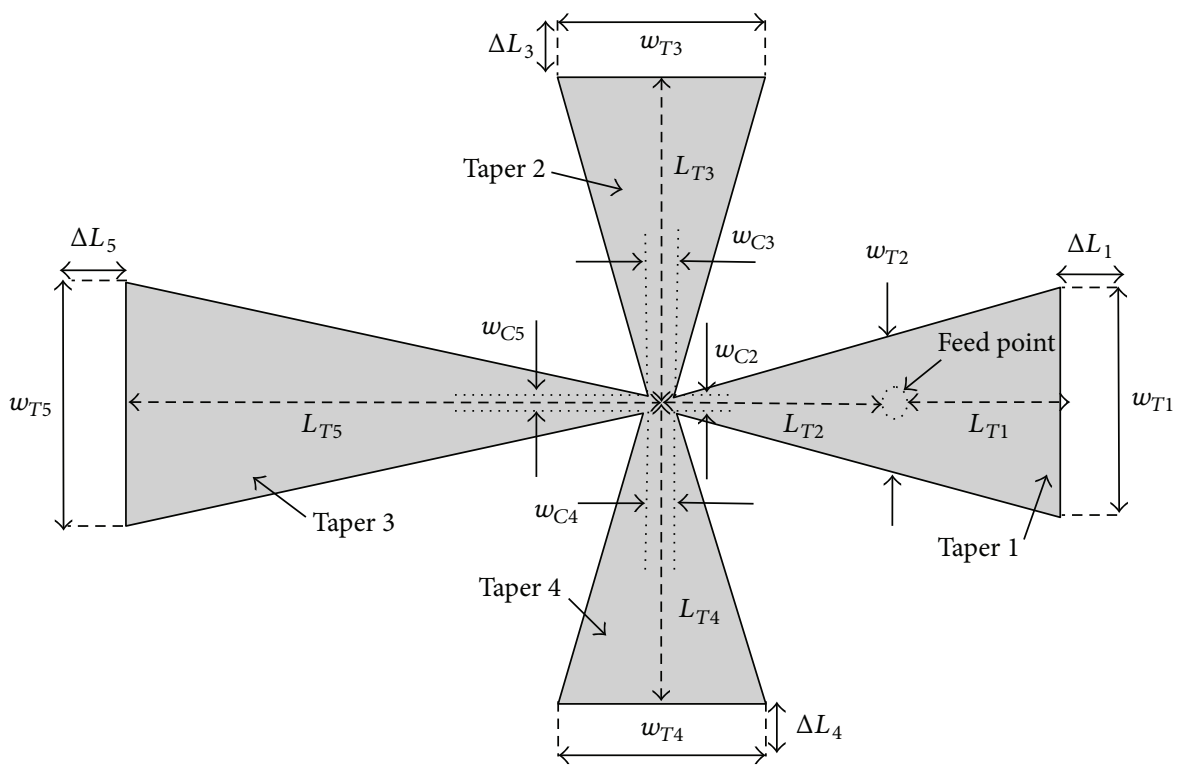

(a)

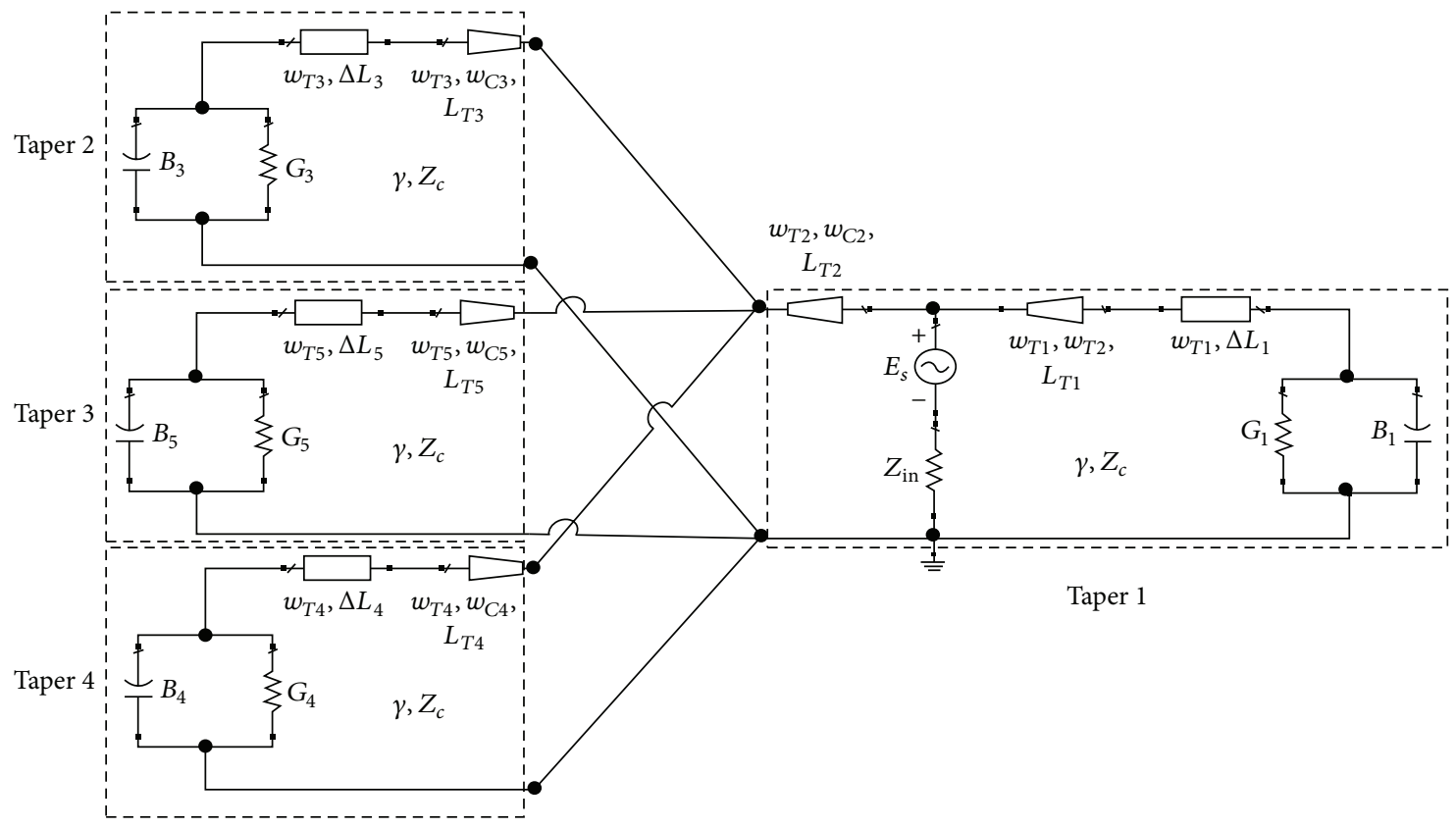

(b)

FIgURE 4: (a) Configuration of the proposed quad-band bowtie antenna. (b) Equivalent circuit model of the proposed quad-band bowtie antenna.

and $4.5 \mathrm{GHz}$ which do not exceed $-15 \mathrm{~dB}$ probably due to the mutual coupling between adjacent elements.

\section{Conclusion}

An accurate equivalent model is developed designated for microstrip bowtie antennas design fed via a coaxial probe. Various antenna configurations, single band, dual-band, and quad-band, have been optimized and designed in this paper. The validity of the equivalent circuit modeling is supported by comparing the values of the bowtie antenna reflection coefficient with those obtained, respectively, with moments method computed by Momentum, the finite integration technique using CST software, and the finite element method of HFSS software. The simulated results show that the desired frequencies used in wireless communication systems, Bluetooth, mobile WiMAX, and two bands of the Unlicensed National Information Infrastructure (U-NII) are efficiently achieved and an excellent coherence between the equivalent circuit model and the moments method and finite integration technique is obtained. It can be concluded that the bowtie antenna is capable of generating multiple resonance modes, 


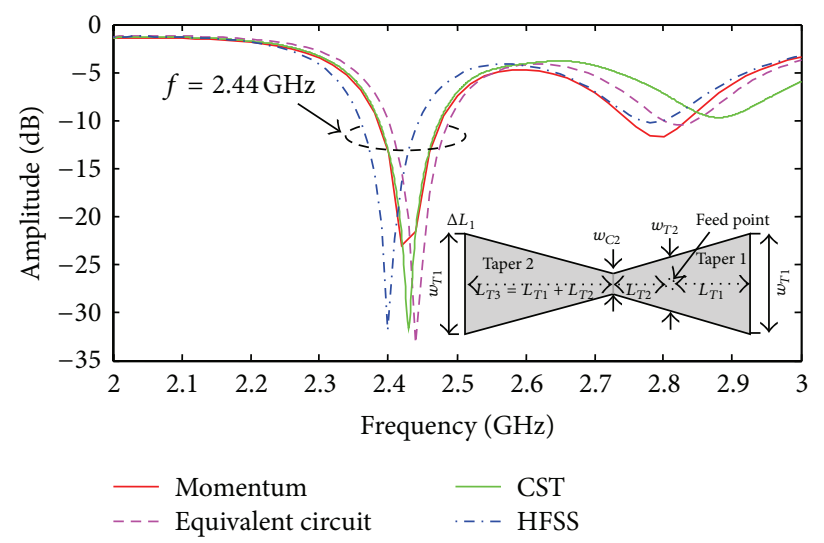

FIGURE 5: Computed return loss of the bowtie antenna designated for WLAN in IEEE $802.11 \mathrm{a} / \mathrm{b}$ applications. Geometry of the bowtie antenna under test is also presented. The antenna parameters are set as $w_{T 1}=24.21 \mathrm{~mm}, w_{\mathrm{C} 2}=0.9 \mathrm{~mm}, L_{T 3}=66.10 \mathrm{~mm}, w_{T 2}=5.49 \mathrm{~mm}, L_{T 1}=$ $53.09 \mathrm{~mm}$, and $L_{T 2}=13.01 \mathrm{~mm}$.

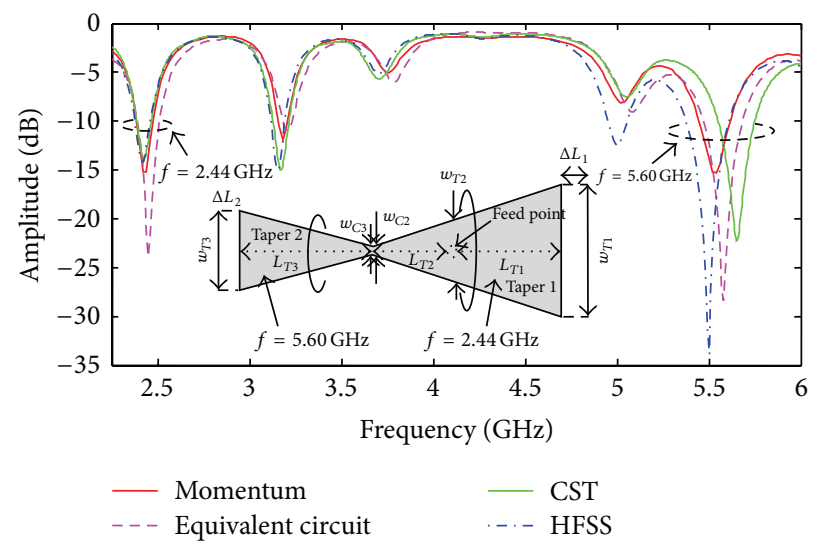

Figure 6: Simulated return loss for the dual-band bowtie antenna and the proposed dual-band bowtie antenna. The antenna parameters are set as $w_{T 3}=28.03 \mathrm{~mm}, w_{C 3}=0.85 \mathrm{~mm}, L_{T 3}=77.08 \mathrm{~mm}, w_{T 1}=17.41 \mathrm{~mm}, w_{T 2}=8.24 \mathrm{~mm}, w_{\mathrm{C} 2}=1.79 \mathrm{~mm}, L_{T 1}=26 \mathrm{~mm}$, and $L_{T 2}=18.29 \mathrm{~mm}$.

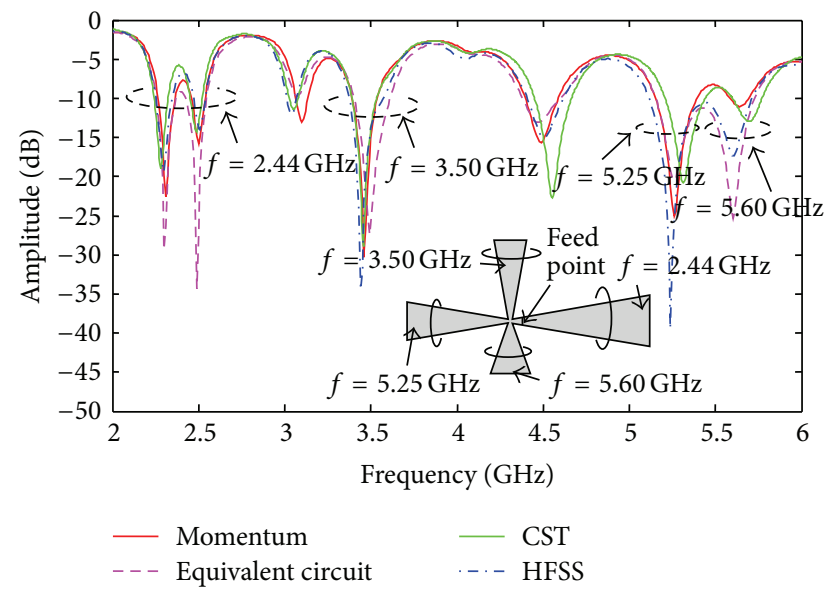

FIGURE 7: Comparison of the simulated return losses for the proposed quad-band bowtie antenna. The geometry of the proposed quad-band bowtie antenna is also displayed. The antenna parameters are set as $w_{T 5}=17.38 \mathrm{~mm}, w_{C 5}=1.62 \mathrm{~mm}, L_{T 5}=89.44 \mathrm{~mm}, w_{T 3}=21.67 \mathrm{~mm}$, $w_{C 3}=1.11 \mathrm{~mm}, L_{T 3}=57.87 \mathrm{~mm}, w_{T 2}=4 \mathrm{~mm}, w_{C 2}=1.78 \mathrm{~mm}, L_{T 2}=6.29 \mathrm{~mm}, w_{T 1}=28.26 \mathrm{~mm}, L_{T 1}=68.84 \mathrm{~mm}, w_{T 4}=20.97 \mathrm{~mm}$, $w_{\mathrm{C} 4}=1.35 \mathrm{~mm}$, and $L_{T 4}=55.67 \mathrm{~mm}$. 
by varying the dimensions and multiplying the number of tapers. The designed antennas can be employed for wireless applications.

\section{Conflict of Interests}

The authors declare that there is no conflict of interests regarding the publication of this paper.

\section{References}

[1] T.-H. Kim and D.-C. Park, "Compact dual-band antenna with double L-slits for WLAN operations," IEEE Antennas and Wireless Propagation Letters, vol. 4, no. 1, pp. 249-252, 2005.

[2] W. Diels, K. Vaesen, P. Wambacq et al., "Single-package integration of RF blocks for a $5 \mathrm{GHz}$ WLAN application," IEEE Transactions on Advanced Packaging, vol. 24, no. 3, pp. 384-391, 2001.

[3] H. Labiod, H. Afifi, and C. Desantis, Bluetooth Zigbee Wifi and Wimax, Springer, 2007.

[4] C. A. Balanis, Modern Antenna Handbook, John Wiley \& Sons, New York, NY, USA, 2008.

[5] M. A. Rahman, M. Hossain, I. S. Iqbal, and S. Sobhan, "Design and performance analysis of a dual-band microstrip patch antenna for mobile WiMAX, WLAN, Wi-Fi and bluetooth applications," in Proceedings of the 3rd International Conference on Informatics, Electronics \& Vision (ICIEV '14), pp. 1-6, Dhaka, Bangladesh, May 2014.

[6] S.-M. Zhang, F.-S. Zhang, W.-M. Li, W.-Z. Li, and H.-Y. Wu, "A multi-band monopole antenna with two different slots for WLAN and WiMAX Applications," Progress in Electromagnetics Research Letters, vol. 28, pp. 173-181, 2012.

[7] Y.-J. Wu, B.-H. Sun, J.-F. Li, and Q.-Z. Liu, "Triple-band omni-directional antenna for WLAN application," Progress in Electromagnetics Research, vol. 76, pp. 477-484, 2007.

[8] Y.-C. Lee and J.-S. Sun, "Compact printed slot antennas for wireless dual- and multi-band operations," Progress in Electromagnetics Research, vol. 88, pp. 289-305, 2008.

[9] I. E. Lager, M. Simeoni, and C. Coman, "Mutual coupling in non-uniform array antennas-an effective recipe," in Proceedings of the 6th European Conference on Antennas and Propagation (EUCAP '12), pp. 1518-1522, IEEE, Prague, Czech, March 2012.

[10] G. A. Casula and P. Maxia, "A multiband printed log-periodic dipole array for wireless communications," International Journal of Antennas and Propagation, vol. 2014, Article ID 646394, 6 pages, 2014.

[11] F. A. Ghaffar, J. R. Bray, and A. Shamim, "Theory and design of a tunable antenna on a partially magnetized ferrite LTCC substrate," IEEE Transactions on Antennas \& Propagation, vol. 62, no. 3, pp. 1238-1245, 2014.

[12] J. J. H. Wang and V. K. Tripp, "Design of multioctave spiralmode microstrip antennas," IEEE Transactions on Antennas \& Propagation, pp. 332-335, 1991.

[13] T. Chio and D. H. Schaubert, "Effects of slotline cavity on dualpolarized tapered slot antenna arrays," in Proceedings of the Antennas and Propagation Society International Symposium, pp. 130-133, IEEE, Orlando, Fla, USA, July 1999.

[14] H. A. Majid, M. K. Abd Rahim, M. R. Hamid, and M. F. Ismail, "Frequency reconfigurable microstrip patch-slot antenna with directional radiation pattern," Progress in Electromagnetics Research, vol. 144, pp. 319-328, 2014.

[15] M. R. Ahsan, M. T. Islam, M. Habib Ullah, H. Arshad, and M. F. Mansor, "Low-cost dielectric substrate for designing low profile multiband monopole microstrip antenna," The Scientific World Journal, vol. 2014, Article ID 183741, 10 pages, 2014.

[16] E. H. van Lil and A. R. van de Capelle, "Transmission line model for mutual coupling between microstrip antennas," IEEE Transactions on Antennas and Propagation, vol. 32, no. 8, pp. 816-821, 1984.

[17] C. A. Balanis, Antenna Theory Analysis and Design, John Wiley \& Sons, 2nd edition, 1997.

[18] S. Didouh, M. Abri, and F. T. Bendimerad, "Corporate-feed multilayer bow-tie antenna array design using a simple transmission line model," Modelling and Simulation in Engineering, vol. 2012, Article ID 327901, 8 pages, 2012. 

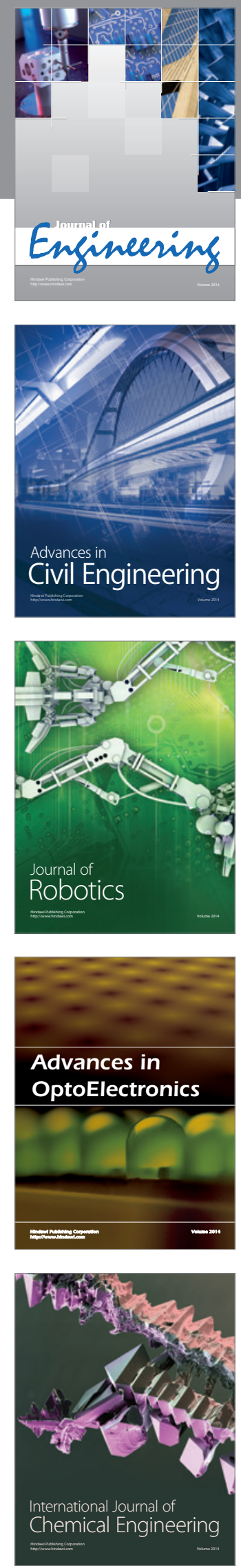

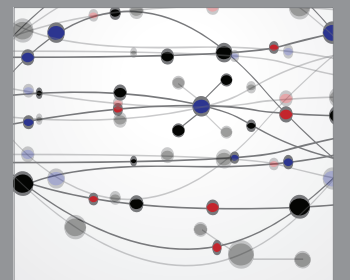

The Scientific World Journal
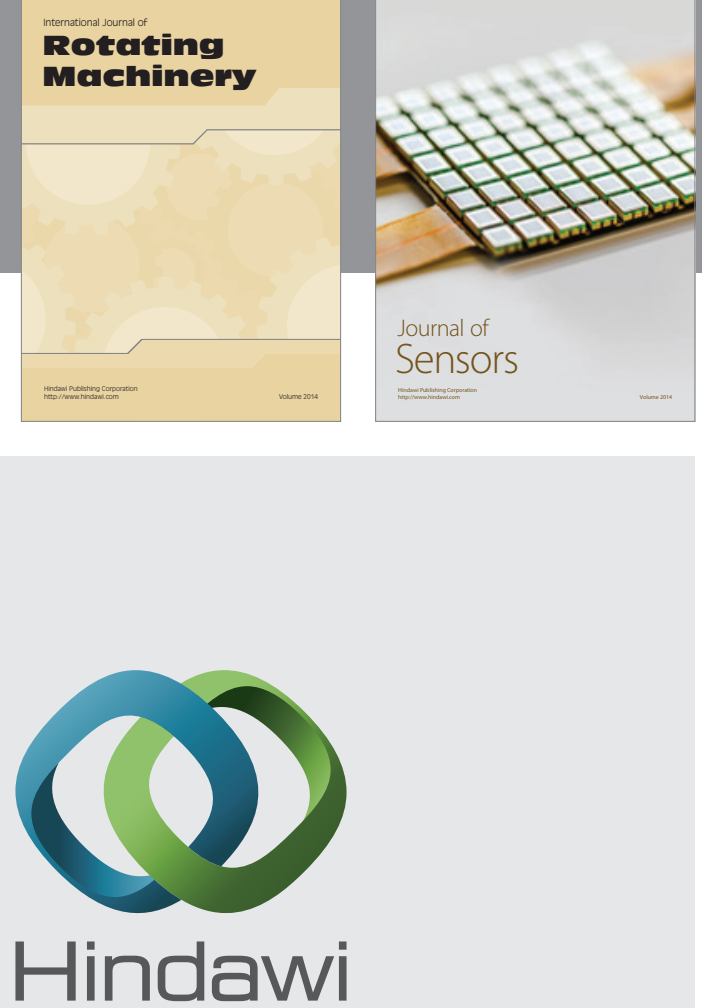

Submit your manuscripts at http://www.hindawi.com
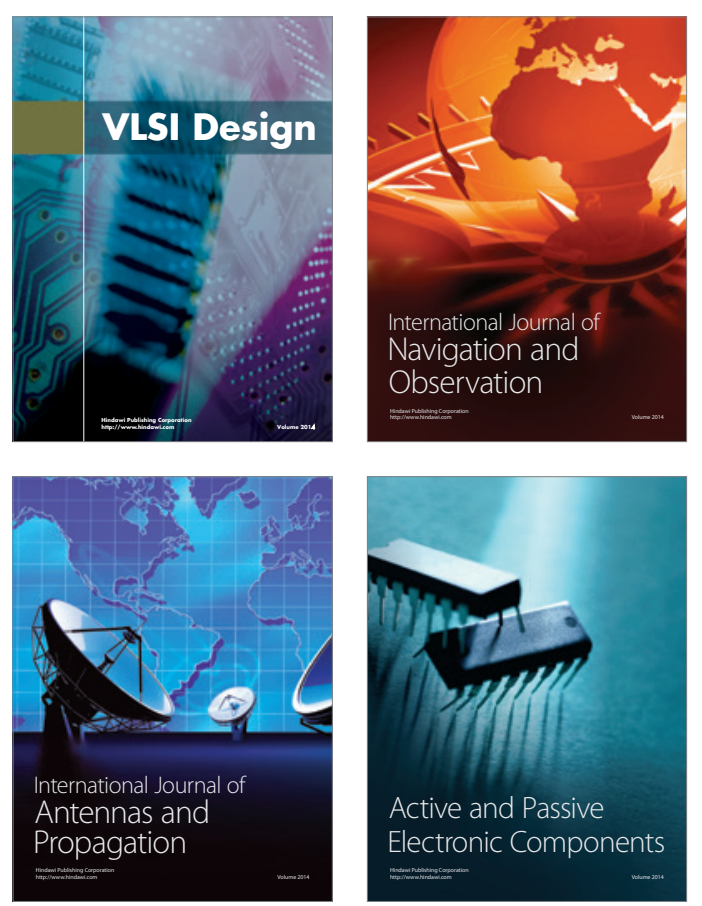
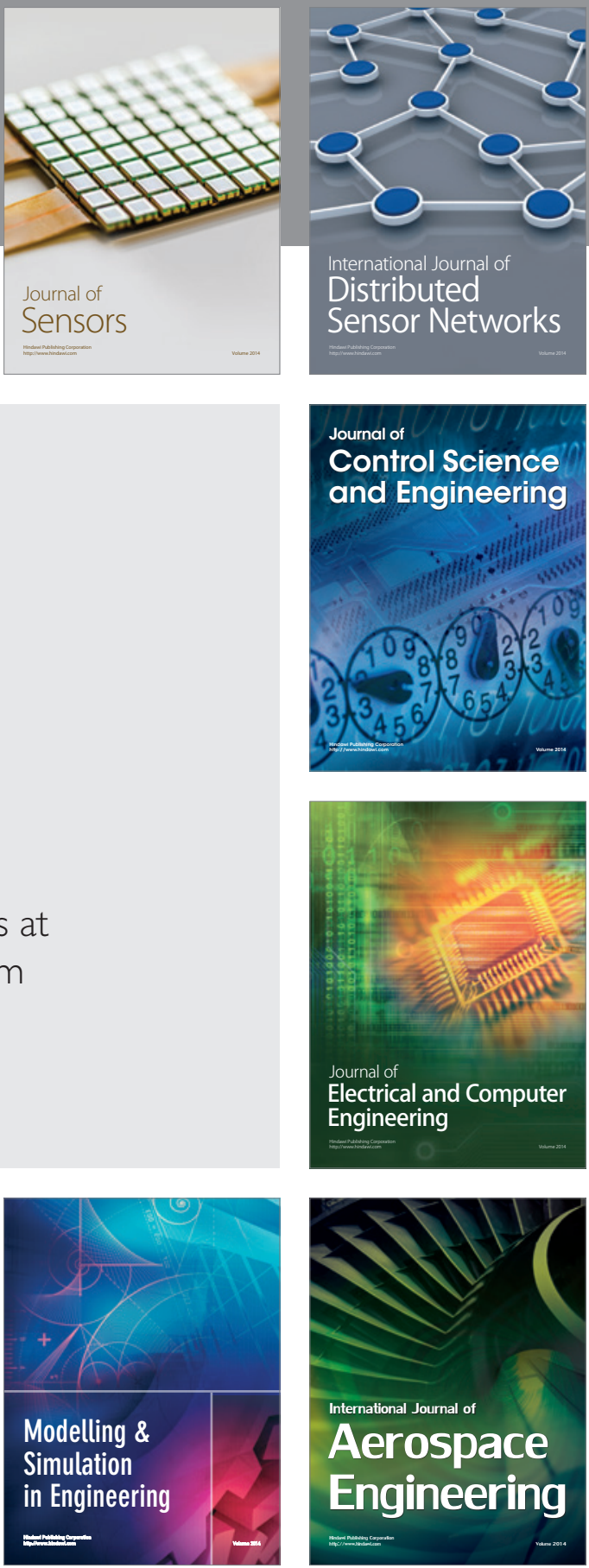

Journal of

Control Science

and Engineering
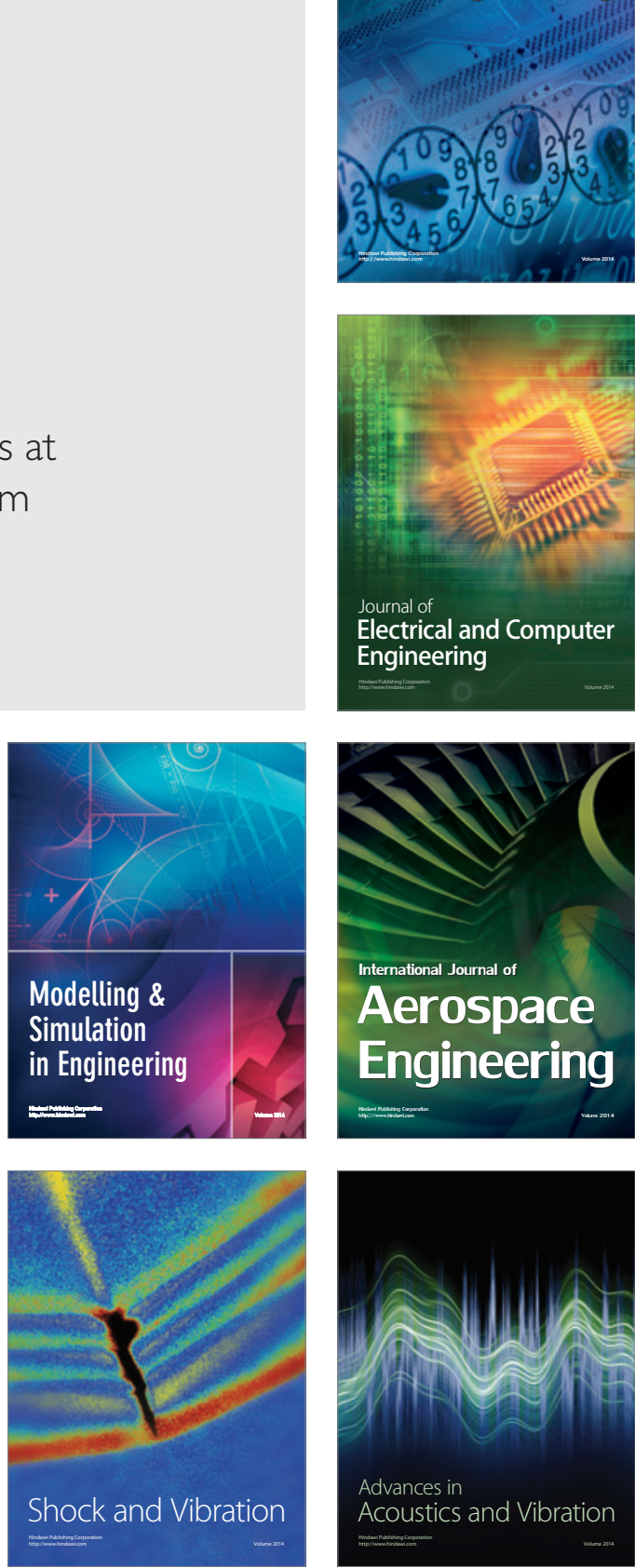